\title{
CHARLES BONNET SYNDROME AND HERPES ENCEPHALITIS
}

Pediatric neurologists at Ondokuz Mayis University, Samsun, Turkey report a 4year-old boy who presented with fever, headache, drowsiness, and seizures. Neurologic exam revealed drowsiness and meningeal signs. Four days following a 3-week course of acyclovir therapy for herpes encephalitis, he developed visual impairment and visual hallucinations lasting 1 week. Loss of vision became total, with normal appearing fundi. Visual hallucinations included unfamiliar children hiding under his bed, and he spoke to someone unknown. The hallucinations occurred 2-3 times daily and were 2-5 minutes in duration, without behavioral changes or cognitive impairment. MRI showed bilateral optic nerve hyperintensity on T2-weighted contrast-enhanced images, consistent with retrobulbar optic neuritis; hyperintensities also involved the dentate nuclei, cerebellum, basal ganglia, and brainstem. EEG showed diffuse slowing and left temporoparietal spike waves. CSF PCR was positive for herpes simplex virus-1 DNA. Following a 3-week course of corticosteroid therapy, the optic neuritis resolved and vision returned to normal after 1 month. During 1 year follow up while taking oxcarbazepine, he had no seizures nor hallucinations. (Aydin OF, Ince H, Tasdemir HA, Ozyurek H. Charles Bonnet Syndrome after herpes simplex encephalitis. Pediatr Neurol 2012 April;46:250-252). (Respond: Dr Aydin, Department of Pediatric Neurology, Faculty of Medicine, Ondokuz Mazyis University, Kurupelit Kampusu, 55139 Samsun, Turkey. E-mail: ofaydin@yahoo.com).

COMMENT. Charles Bonnet syndrome (CBS) is characterized by complex visual hallucinations in association with visual impairment and normal cognitive and behavioral status. The syndrome is described usually in adults with macular degeneration and sometimes with multiple sclerosis. In a 9-year-old partially sighted boy with paroxysmal visual zoopsias the $\mathrm{CB}$ syndrome is described as phantom vision (Mewasingh LD et al. Pediatr Neurol 2002 Feb;26(2):143-145). Only 3 previous pediatric cases in the literature were cited, two with cone-rod dystrophy. In these children, 6 and 8 years old, formed visual hallucinations noted shortly after loss of vision included geometric shapes, people, and buildings, stationary and in motion. (Schwartz TL, Vahgei L. Charles Bonnet syndrome in children. J AAPOS 1998 Oct;2(5):310-313).

CBS should be differentiated from migraine and epilepsy. One report of a case of CBS disappearing with successive monotherapies of carbamazepine and valproic acid but worsening with levetiracetam is suggestive of an epilepsy origin for CBS (Segers K. Acta Neurol Belg 2009 Mar;109(1):42-43).

Epilepsy and complex visual hallucinations. Except for one case, a 12-year-old boy with birth trauma and seizures who described seeing colored triangles followed by seeing a "robber coming after him with a gun," Penfield reported no elaborate visual hallucinations elicited by stimulation of the occipital lobes, only gross light, shadows and colors. (Penfield W, Jasper HH. 1954).

Lennox WG reports a girl, aged 9 years, who had generalized tonic-clonic seizures, preceded by visual hallucinations in which small objects or persons, "like a comic book," appeared in one field of vision, and followed by headache or vomiting. The EEG showed triphasic spikes or slow spike-and-wave discharges in the right posterior temporal or occipital area. The father had migraine from childhood and a maternal aunt 
had complex partial seizures. This case points to both a migraine and seizure origin for the visual hallucinations. (Lennox WG. Epilepsy and Related Disorders. Boston: Little, Brown, 1960; Vol 1, p 270). EEG is recommended if CBS develops in a patient with worsening of neurological signs (Ossola M et al. Epileptic mechanisms in Charles Bonnet syndrome. Epilepsy Behav 2010 May;18(1-2):119-122).

\section{SEIZURE DISORDERS}

\section{ICTAL EPILEPTIC HEADACHE WITH IDIOPATHIC EPILEPSY}

Neurologists at the University of Rome, Italy report a 37-year-old woman with drug-resistant generalized epilepsy and headache who had a sudden headache during a 24-h EEG that displayed epileptic activity. Generalized S/W discharges and polyspike and wave discharges persisted for $60 \mathrm{~min}$ until the headache disappeared. The case represents a rare example of ictal epileptic headache in generalized idiopathic epilepsy. (Fanella M, Fattouch J, Casciato S, et al. Ictal epileptic headache as "subtle" symptom in generalized idiopathic epilepsy. Epilepsia 2012 March;53(4):e67-e70). (Respond: Dr Carlo Di Bonaventura, Department of Neuroscience, Neurology Unit, "Sapienza" University of Rome, Viale dell'Universita 30, 00185 Rome, Italy. E-mail: c_dibonaventura@yahoo.it).

COMMENT. In the authors' opinion, "ictal epileptic headache" warrants listing in the international classification of both epilepsy and headache. This case report is a rare example of the entity.

\section{RISK OF EPILEPSY AFTER FEBRILE SEIZURES}

Investigators at the Institute of Neurology, London, and at other centers in the UK and the Netherlands conducted a prospective follow-up of 181 infants from the onset of febrile seizures for a median of 21.6 years, to estimate the long-term risk of developing epilepsy. Of these, 175 (97\%) were seizure-free in the preceding 5 years, and $171(94 \%)$ were seizure-free and off antiepileptic drugs. Six percent developed epilepsy. In total, 17 (7.7\%) had afebrile seizures, of whom $14(6.4 \%)$ had 2 or more afebrile seizures (epilepsy). The mean time to the second afebrile seizure was 5.7 years. At 20 years after the index febrile seizure, $6.7 \%$ had developed epilepsy. The risk of developing epilepsy in the cohort over the whole follow-up period was 10 times that of the general population. The standardized incidence ratio was significantly elevated in the 0- to 14-year age groups but not in the 15- to 19-year age group. The risk of developing epilepsy in people who had febrile seizures appears to decrease with time. A history of 4 or more febrile seizures is a risk factor for development of epilepsy. (Neligan A, Bell GS, Giavasi C, et al. Long-term risk of developing epilepsy after febrile seizures. A prospective cohort study. Neurology 2012 April 10;78:1166-1170), (Response and reprints: Prof Sander. Email: I.sander@ucl.ac.uk).

COMMENT. In this study, no differentiation was made between simple and complex febrile seizures. The association between febrile seizures and later epilepsy is 